\title{
GREEN'S FUNCTIONS OF THE WAVE EQUATION IN DIFFERENT DIMENSIONS
}

\author{
MOHAMMAD KHORRAMI
}

Received 01 April, 2019

\begin{abstract}
Relations of different Green's functions of the wave equation in different dimensions, with each other and with the Green's function of the Laplace equation, are investigated. These are used to obtain the Green's functions of the wave equation in different dimensions.
\end{abstract}

2010 Mathematics Subject Classification: 35J08; 35L05; 35J05

Keywords: Green's function, wave equation, Laplace equation, different dimensions

\section{INTRODUCTION}

The simple wave equation is a hyperbolic partial differential equation (see [12] for example). This equation arises in many problems. Among these are the vibrations of a string, the vibrations of a membrane, the propagation of sound in fluids ([5-7] respectively, for example). Another important example is the propagation of light, or electromagnetic field [4]. All of these phenomena have one thing in common, and that thing is propagation with finite speed. In more technical terms, the effect of a point source is not propagated outside the wave cone (light cone in the case of light [10]).

As the wave equation is linear, one way to investigate the effect of a point source on the wave is to study the Green's function [1]. One important question is whether the effect of point source is propagated only on the wave cone or on the wave cone and inside the wave cone as well. One can also ask whether this behaviour depends on the dimension of the space or not.

The wave equation is a hyperbolic equation, as mentioned before. But if one performs an analytic continuation on the time variable, it is seen that for imaginary time the wave equation is transformed to the Laplace equation, [13]. The Laplace equation arises in many cases as well, including the problem of finding the electrostatic potential $[2,8,9]$. While the wave equation is hyperbolic, the Laplace equation is elliptic and the properties of the solutions of the wave equation and the Laplace equation are totally different. Yet there arises a question on the possible relation of a solution of the Laplace equation with a solution of the wave with imaginary time coordinate. To 
be more specific, is it possible to obtain the Green's function of the wave equation by performing an analytic continuation on the Green's function of the Laplace equation?

Finally, the qualitative behaviour of the Green's functions of the Laplace equation in different dimensions are almost similar, specially if the dimension of the space is larger than two. Is this the case for the Green's function of the wave equation as well?

The aim of this paper is to address these questions. It is shown that the Green's function of the wave equation is related to that of the Laplace equation through an analytic continuation. But this is the case only for a certain kind of the Green's functions of the wave equation, which is the Feynman Green's function. This kind of the Green's function does not arise very often in the context of classical physics, but it plays a crucial role in quantum physics [3]. Yet it is possible to express the retarded and advanced Green's function (which arise more often in classical physics) in terms of the Feynman Green's function. Using these, various kinds of the Green's function of the wave equation in different dimensions are obtained. As a result, it is seen that for odd space dimensions (except for the one dimensional space) the effect of a point source is propagated only on the wave cone, while for even dimensional spaces propagation takes place inside the wave cone as well.

The scheme of the paper is the following. In Section 2 the wave equation and the equation governing its Green's function are introduced. In Section 3 various kinds of the Green's function of the wave equation are discussed. In Section 4 the relations between these Green's functions are studied. In Section 5 a recursive relation in terms of the dimension of the space is presented for the Green's function of the wave equation. In Section 6 the Feynman Green's function is obtained as an analytic continuation of the Green's function of the Laplace equation. Using that, other Green's functions of the wave equation are obtained as well. Section 7 is devoted to concluding remarks.

\section{THE WAVE EQUATION AND ITS GREEN'S FUNCTION}

The (homogeneous) simple wave equation is

$$
\left(-\frac{\partial^{2}}{\partial t^{2}}+\nabla^{2}\right) \psi(t, r)=0
$$

where $(t, \boldsymbol{r})$ is a point in the space-time, and the units are chosen so that the speed of the wave is unity. The general solution of this equation is an arbitrary linear combination of plain waves $\exp (-\mathrm{i} \omega t+\mathrm{i} \boldsymbol{k} \cdot \boldsymbol{r})$, where $\omega$ and $\boldsymbol{k}$ satisfy the dispersion relation

$$
\omega^{2}-\boldsymbol{k} \cdot \boldsymbol{k}=0 \text {. }
$$

The wave equation with source is

$$
\left(-\frac{\partial^{2}}{\partial t^{2}}+\nabla^{2}\right) \psi(t, \boldsymbol{r})=j(t, \boldsymbol{r})
$$


where $j$ (the source) is an arbitrary function of the space-time. As this equation is linear, its solution is

$$
\psi(t, \boldsymbol{r})=\int \mathrm{d} t \mathrm{~d} V G\left(t, \boldsymbol{r} ; t^{\prime}, \boldsymbol{r}^{\prime}\right) j\left(t^{\prime}, \boldsymbol{r}^{\prime}\right)+\psi_{\mathrm{h}}(t, \boldsymbol{r}) .
$$

$\psi_{\mathrm{h}}$ is an arbitrary function satisfying the homogeneous wave equation (2.1), and $G$ is the Green's function of the wave equation, satisfying

$$
\left(-\frac{\partial^{2}}{\partial t^{2}}+\nabla^{2}\right) G\left(t, \boldsymbol{r} ; t^{\prime}, \boldsymbol{r}^{\prime}\right)=\delta\left(t-t^{\prime}\right) \delta^{D}\left(\boldsymbol{r}-\boldsymbol{r}^{\prime}\right)
$$

$D$ is the dimension of the space. The wave equation is space-time-independent, in the sense that in the differential operator in the left-hand side, there are no explicit functions of space-time. This results in the fact that one can choose $G$ to be a function of the difference of the primed and unprimed variables:

$$
G\left(t, r ; t^{\prime}, \boldsymbol{r}^{\prime}\right)=G\left(t-t^{\prime}, \boldsymbol{r}-\boldsymbol{r}^{\prime}\right) \text {. }
$$

Using this, equation (2.5) is rewritten as

$$
\left(-\frac{\partial^{2}}{\partial t^{2}}+\nabla^{2}\right) G(t, \boldsymbol{r})=\delta(t) \delta^{D}(\boldsymbol{r})
$$

\section{DifFERENT GREEN'S FUNCTIONS OF THE WAVE EQUATION}

The solution to (2.5) or (2.7) is not unique. In fact, adding any solution of the homogenous equation (2.1) to an arbitrary solution of (2.7), another solution of (2.7) is obtained. Another way to see this, is using the Fourier transformation. The Fourier transform of (2.7) is

$$
\left(\omega^{2}-\boldsymbol{k} \cdot \boldsymbol{k}\right) \tilde{G}(\omega, \boldsymbol{k})=1 .
$$

The solution to this is not unique: Any function of the form

$$
\tilde{G}_{\mathrm{h}}=f(\omega, \boldsymbol{k}) \delta\left(\omega^{2}-\boldsymbol{k} \cdot \boldsymbol{k}\right)
$$

satisfies the homogenous equation

$$
\left(\omega^{2}-\boldsymbol{k} \cdot \boldsymbol{k}\right) \tilde{G}_{\mathrm{h}}(\omega, \boldsymbol{k})=0,
$$

where $f$ is an arbitrary function. The meaning of (3.2) is that $G_{\mathrm{h}}$ is a superposition of plane waves, satisfying the dispersion relation (2.2). So adding such a function $\tilde{G}_{\mathrm{h}}$ to a solution of (3.1), gives another solution of (3.1). Another point (of course related to this) is that to obtain the Green's function from its Fourier transform, this Fourier transform should be rigorously defined. The solution to (3.1) is

$$
\tilde{G}(\omega, \boldsymbol{k})=\operatorname{pf} \frac{1}{\omega^{2}-\boldsymbol{k} \cdot \boldsymbol{k}}+\tilde{G}_{\mathrm{h}}(\omega, \boldsymbol{k}),
$$

where $\operatorname{pf}(1 / x)$ is a distribution the effect of which on the test function $\phi$ is [11]

$$
\langle\operatorname{pf}(1 / x), \phi(x)\rangle=\lim _{\varepsilon \rightarrow 0^{+}}\left(\int_{-\infty}^{-\varepsilon}+\int_{\varepsilon}^{\infty}\right) \frac{\mathrm{d} x \phi(x)}{x} .
$$


Three special choices for $\tilde{G}$ are the retarded Green's function:

$$
\tilde{G}_{\mathrm{R}}=\frac{1}{(\omega+\mathrm{i} \varepsilon)^{2}-\boldsymbol{k} \cdot \boldsymbol{k}}
$$

the advanced Green's function:

$$
\tilde{G}_{\mathrm{A}}=\frac{1}{(\omega-\mathrm{i} \varepsilon)^{2}-\boldsymbol{k} \cdot \boldsymbol{k}},
$$

and the Feynman Green's function:

$$
\tilde{G}_{\mathrm{F}}=\frac{1}{\omega^{2}-\boldsymbol{k} \cdot \boldsymbol{k}+\mathrm{i} \varepsilon} .
$$

In all cases, the limit $\varepsilon \rightarrow 0^{+}$is meant. The relation

$$
G(t, \boldsymbol{r})=\frac{1}{(2 \pi)^{D+1}} \int \mathrm{d} \omega \mathrm{d}^{D} k \tilde{G}(\omega, \boldsymbol{k}) \exp (-\mathrm{i} \omega t+\mathrm{i} \boldsymbol{k} \cdot \boldsymbol{r})
$$

relates each of these Green's functions to its Fourier transform. The integral over $\omega$ can be transformed (for $t>0$ and $t<0$ ) to an integral over a closed path on the complex $\omega$ plane:

$$
G(t, \boldsymbol{r})=\frac{1}{(2 \pi)^{D+1}} \int \mathrm{d}^{D} k \int_{S_{ \pm}} \mathrm{d} \omega \tilde{G}(\omega, \boldsymbol{k}) \exp (-\mathrm{i} \omega t+\mathrm{i} \boldsymbol{k} \cdot \boldsymbol{r}) \quad \pm t>0 .
$$

$S_{+}$is a path beginning at $\omega \rightarrow-\infty$, going on the real line to $\omega \rightarrow+\infty$, and returning on an infinite semicircle in the half-plane $\operatorname{Im}(\omega)<0$ to $\omega \rightarrow-\infty$. $S_{-}$is a path beginning at $\omega \rightarrow-\infty$, going on the real line to $\omega \rightarrow+\infty$, and returning on an infinite semicircle in the half-plane $\operatorname{Im}(\omega)>0$ to $\omega \rightarrow-\infty$. This shows that

$$
G_{\mathrm{R}}=0, \quad t<0,
$$

and

$$
G_{\mathrm{A}}=0, \quad t>0
$$

as in each case the integrand as a function of $\omega$ is analytic inside the integration contour. The reason for calling these Green's functions retarded and advanced, is that the retarded Green's function is nonzero only for $t>0$, while the advanced Green's function is nonzero only for $t<0$. In the case of the Feynman Green's function, it is seen that in $t>0$ only terms with positive frequency $(\omega)$ contribute, while for $t<0$ only terms with negative frequency $(\omega)$ contribute.

Consider two auxiliary functions $G_{+}$and $G_{-}$. Their definition is similar to (3.9), except that the integration path in the complex $\omega$ plane is not the real line:

$$
G_{ \pm}(t, \boldsymbol{r})=\frac{1}{(2 \pi)^{D+1}} \int \mathrm{d}^{D} k \oint_{C_{ \pm}} \mathrm{d} \omega \tilde{G}(\omega, \boldsymbol{k}) \exp (-\mathrm{i} \omega t+\mathrm{i} \boldsymbol{k} \cdot \boldsymbol{r}) .
$$

$C_{+}$is a contour going counter-clockwise so that the point $\omega=(\boldsymbol{k} \cdot \boldsymbol{k})^{1 / 2}$ is inside it and the point $\omega=-(\boldsymbol{k} \cdot \boldsymbol{k})^{1 / 2}$ is outside it. $C_{-}$is a contour going counter-clockwise so that the point $\omega=-(\boldsymbol{k} \cdot \boldsymbol{k})^{1 / 2}$ is inside it and the point $\omega=(\boldsymbol{k} \cdot \boldsymbol{k})^{1 / 2}$ is outside it. 
These functions don't satisfy (2.7) but, as will be seen, the retarded, advanced, and Feynman Green's functions can be written in terms of them.

\section{RELATION OF DIFFERENT GREEN'S FUNCTIONS WITH EACH OTHER}

From the definitions of the previous section, it is seen that

$$
\begin{aligned}
& G_{\mathrm{R}}(t, \boldsymbol{r})=-\theta(t)\left[G_{+}(t, \boldsymbol{r})+G_{-}(t, \boldsymbol{r})\right], \\
& G_{\mathrm{A}}(t, \boldsymbol{r})=\theta(-t)\left[G_{+}(t, \boldsymbol{r})+G_{-}(t, \boldsymbol{r})\right], \\
& G_{\mathrm{F}}(t, \boldsymbol{r})=-\theta(t) G_{+}(t, \boldsymbol{r})+\theta(-t) G_{-}(t, \boldsymbol{r}) .
\end{aligned}
$$

Using (3.13), one can relate $G_{+}$and $G_{-}$to each other and their complex conjugates. One has

$$
G_{+}(-t, \boldsymbol{r})=-G_{-}(t, \boldsymbol{r}) .
$$

To obtain this, one uses the change of variable $z=-\omega$. The integration contour for the variable $z$ is $C_{-}$, every point of which is the negative of a point on $C_{+}$. One also has

$$
G_{+}(-t, \boldsymbol{r})=-G_{+}^{*}(t, \boldsymbol{r}) .
$$

To obtain this, the change of variable $s=\omega^{*}$ has been used. The integration contour for the variable $s$ is a contour every point of which is the complex conjugate of a point on $C_{+}$. This is the same as $C_{+}$, but going clockwise. So the integral on this contour is the negative of the integral on $C_{+}$. The final result is

$$
\begin{aligned}
& G_{+}(-t, \boldsymbol{r})=-G_{-}(t, \boldsymbol{r})=-G_{+}^{*}(t, \boldsymbol{r}), \\
& G_{-}(-t, \boldsymbol{r})=-G_{+}(t, \boldsymbol{r})=-G_{-}^{*}(t, \boldsymbol{r}) .
\end{aligned}
$$

Using these and the relations (4.1), all of these functions can be written in terms of the Feynman Green's function:

$$
\begin{aligned}
& G_{+}(t, \boldsymbol{r})=-\theta(t) G_{\mathrm{F}}(t, \boldsymbol{r})+\theta(-t) G_{\mathrm{F}}^{*}(t, \boldsymbol{r}), \\
& G_{-}(t, \boldsymbol{r})=-\theta(t) G_{\mathrm{F}}^{*}(t, \boldsymbol{r})+\theta(-t) G_{\mathrm{F}}(t, \boldsymbol{r}), \\
& G_{\mathrm{R}}(t, \boldsymbol{r})=\theta(t)\left[G_{\mathrm{F}}(t, \boldsymbol{r})+G_{\mathrm{F}}^{*}(t, \boldsymbol{r})\right], \\
& G_{\mathrm{A}}(t, \boldsymbol{r})=\theta(-t)\left[G_{\mathrm{F}}(t, \boldsymbol{r})+G_{\mathrm{F}}^{*}(t, \boldsymbol{r})\right] .
\end{aligned}
$$

So, knowing the Feynman Green's function one can obtain the other four functions.

\section{RECURSION RELATION FOR THE GREEN'S FUNCTION IN TERMS OF THE DIMENSION}

The general form of the five functions introduced is

$$
G(t, \boldsymbol{r})=\frac{1}{(2 \pi)^{D+1}} \int \mathrm{d}^{D} k \int_{C} \mathrm{~d} \omega \frac{1}{\omega^{2}-\boldsymbol{k} \cdot \boldsymbol{k}} \exp (-\mathrm{i} \omega t+\mathrm{i} \boldsymbol{k} \cdot \boldsymbol{r}) .
$$


The integral on the right-hand side depends on the integration contour $C$. The five functions introduced in the previous section correspond to five different contours. The above expression can be written as

$$
\begin{aligned}
G^{(D)}(t, \boldsymbol{r})= & \frac{S_{D-2}}{(2 \pi)^{D+1}} \int_{0}^{\infty} k^{D-1} \mathrm{~d} k \int_{0}^{\pi} \sin ^{D-2} \theta \mathrm{d} \theta \\
& \times \int_{C} \mathrm{~d} \omega \frac{1}{\omega^{2}-k^{2}} \exp (-\mathrm{i} \omega t+\mathrm{i} k r \cos \theta) .
\end{aligned}
$$

$S_{D}$ is the area of a $D$-dimensional sphere of radius one. To arrive at this result, use has been made of the fact that the contour $C$ depends on only the length of $k$. Differentiating the above expression with respect to $r$, one obtains

$$
\begin{aligned}
\frac{\partial}{\partial r} G^{(D)}(t, \boldsymbol{r})= & \frac{S_{D-2}}{(2 \pi)^{D+1}} \int_{0}^{\infty} k^{D-1} \mathrm{~d} k \int_{0}^{\pi} \sin ^{D-2} \theta \mathrm{d} \theta \\
& \times \int_{C} \mathrm{~d} \omega \frac{1}{\omega^{2}-k^{2}} \exp (-\mathrm{i} \omega t+\mathrm{i} k r \cos \theta) \mathrm{i} k \cos \theta .
\end{aligned}
$$

Integrating by parts on $\theta$ results in

$$
\begin{aligned}
\frac{\partial}{\partial r} G^{(D)}(t, \boldsymbol{r})= & \frac{S_{D-2}}{(2 \pi)^{D+1}} \int_{0}^{\infty} k^{D-1} \mathrm{~d} k \int_{0}^{\pi} \sin ^{D-2} \theta \mathrm{d} \theta \\
& \times \int_{C} \mathrm{~d} \omega \frac{1}{\omega^{2}-k^{2}} \exp (-\mathrm{i} \omega t+\mathrm{i} k r \cos \theta) \frac{-k^{2} r \sin ^{2} \theta}{D-1} .
\end{aligned}
$$

From this,

$$
\frac{\partial}{r \partial r} G^{(D)}(t, \boldsymbol{r})=\frac{-4 \pi^{2} S_{D-2}}{(D-1) S_{D}} G^{(D+2)}(t, \boldsymbol{r}) .
$$

Substituting

$$
S_{D}=\frac{2 \pi^{(D+1) / 2}}{\Gamma[(D+1) / 2]},
$$

one obtains

$$
G^{(D+2)}(t, \boldsymbol{r})=-\frac{1}{\pi} \frac{\partial}{\partial r^{2}} G^{(D)}(t, \boldsymbol{r}) .
$$

This relation is true for all of the five functions introduced. It is, however, assumed that $D \geq 1$. Otherwise there is no integration on $k$. In fact for $D=0$, there is no space for the wave to propagate in.

\section{Determination of the Feynman Green's Function from the GREEN'S FUNCTION OF THE LAPLACE EQUATION}

The Green's function of the Laplace equation in $D+1$ dimensions satisfy

$$
\left(\frac{\partial}{\partial t^{2}}+\nabla^{2}\right) G_{\mathrm{E}}(t, \boldsymbol{r})=\delta(t) \delta^{D}(\boldsymbol{r}) .
$$


This is the analogue of (2.7) for the Green's function of the wave equation. One obtains

$$
G_{\mathrm{E}}(t, \boldsymbol{r})=\frac{1}{(2 \pi)^{D+1}} \int \mathrm{d}^{D} k \int \mathrm{d} \omega \frac{1}{-\omega^{2}-\boldsymbol{k} \cdot \boldsymbol{k}} \exp (-\mathrm{i} \omega t+\mathrm{i} \boldsymbol{k} \cdot \boldsymbol{r}) .
$$

Integrating over $\omega$,

$$
G_{\mathrm{E}}(t, \boldsymbol{r})=\frac{-1}{(2 \pi)^{D}} \int \mathrm{d}^{D} k \frac{\theta(t) e^{-k t}+\theta(-t) e^{k t}}{2 k} \exp (\mathrm{i} \boldsymbol{k} \cdot \boldsymbol{r}) .
$$

Using (3.8) and (3.9) for the Feynman Green's function,

$$
G_{\mathrm{F}}(t, \boldsymbol{r})=\frac{-i}{(2 \pi)^{D}} \int \mathrm{d}^{D} k \frac{\theta(t) e^{-\mathrm{i} k t}+\theta(-t) e^{\mathrm{i} k t}}{2 k} \exp (\mathrm{i} \boldsymbol{k} \cdot \boldsymbol{r}) .
$$

Compare it with (6.3). (6.3) is for real $t$. But one can analytically continuate (6.3) to complex $t$ with nonzero real part:

$$
G_{\mathrm{E}}(t, \boldsymbol{r})=\frac{-1}{(2 \pi)^{D}} \int \mathrm{d}^{D} k \frac{\theta[\operatorname{Re}(t)] e^{-k t}+\theta[-\operatorname{Re}(t)] e^{k t}}{2 k} \exp (\mathrm{i} \boldsymbol{k} \cdot \boldsymbol{r}) .
$$

It is seen that this function is analytic for $\operatorname{Re}(t) \neq 0$. Now compare (6.4) and (6.5). It is seen that for real $t$,

$$
G_{\mathrm{F}}(t, \boldsymbol{r})=\mathrm{i} \lim _{\varepsilon \rightarrow 0^{+}} G_{\mathrm{E}}\left(\mathrm{i} t e^{-\mathrm{i} \varepsilon}, \boldsymbol{r}\right) .
$$

Note that the real part of $\xi:=\mathrm{i} e^{-\mathrm{i} \varepsilon}$ is positive (for positive $\varepsilon$ ). So for real $t$,

$$
\theta[\operatorname{Re}(\xi t)]=\theta(t) .
$$

To arrive at (6.6), (6.7) has been used.

So, to obtain the Feynman Green's function in a $D+1$ dimensional spacetime, it is sufficient to know the Green's function of the Laplace equation in $D+1$ dimensions. For $D>1$, it is

$$
G_{\mathrm{E}}(t, \boldsymbol{r})=\frac{R^{1-D}}{(1-D) S_{D}},
$$

where

$$
R:=\sqrt{t^{2}+\boldsymbol{r} \cdot \boldsymbol{r}} .
$$

To arrive at (6.8), one uses a spherically symmetric ansatz for $G_{\mathrm{E}}$ and applies the Gauss' theorem. (6.6) and (6.8) result in

$$
G_{\mathrm{F}}(t, \boldsymbol{r})=\mathrm{i} \frac{\left(r^{2}-t^{2}+\mathrm{i} \varepsilon\right)^{(1-D) / 2}}{(1-D) S_{D}} .
$$

( $2 \varepsilon t^{2}$ has been substituted with $\varepsilon$.) It is seen that this expression satisfies (5.7).

Using this function, one can obtain the other four functions. To do so, one way is to determine the real and imaginary parts of the Feynman Green's function. From 
now on, one should treat even and odd $D$ 's separately. For even $D$ 's, $(D-1) / 2$ is half integer and

$$
\operatorname{Re}\left[G_{\mathrm{F}}(t, \boldsymbol{r})\right]=-(-1)^{D / 2} \operatorname{pf}\left[\frac{\left(t^{2}-r^{2}\right)^{(1-D) / 2}}{(1-D) S_{D}} \theta\left(t^{2}-r^{2}\right)\right], \quad(D / 2) \in \mathbb{Z},
$$

and

$$
\operatorname{Im}\left[G_{\mathrm{F}}(t, \boldsymbol{r})\right]=\operatorname{pf}\left[\frac{\left(r^{2}-t^{2}\right)^{(1-D) / 2}}{(1-D) S_{D}} \theta\left(r^{2}-t^{2}\right)\right], \quad(D / 2) \in \mathbb{Z} .
$$

In these relations, the action of $\mathrm{pf}\left[x^{-n-(1 / 2)} \theta(x)\right]$ on the test function $\phi$ has been defined through the recursion relation

$$
\int \mathrm{d} x \operatorname{pf}\left[x^{-n-1-(1 / 2)} \theta(x)\right] \phi(x):=\frac{1}{n+(1 / 2)} \int \mathrm{d} x \operatorname{pf}\left[x^{-n-(1 / 2)} \theta(x)\right] \phi^{\prime}(x) .
$$

$x^{-(1 / 2)} \theta(x)$ is locally integrable and the sign pf in front of it has no effect.

For odd $D$ 's, $(D-1) / 2$ is integer and

$$
\operatorname{Re}\left[G_{\mathrm{F}}(t, \boldsymbol{r})\right]=-\frac{1}{4 \pi^{(D-1) / 2}} \delta^{[(D-3) / 2]}\left(t^{2}-r^{2}\right), \quad[(D-1) / 2] \in \mathbb{Z},
$$

and

$$
\operatorname{Im}\left[G_{\mathrm{F}}(t, \boldsymbol{r})\right]=\operatorname{pf}\left[\frac{\left(r^{2}-t^{2}\right)^{(1-D) / 2}}{(1-D) S_{D}}\right], \quad[(D-1) / 2] \in \mathbb{Z} .
$$

To obtain these relations, use has been made of

$$
\frac{1}{x+\mathrm{i} \varepsilon}=\mathrm{pf} \frac{1}{x}-\mathrm{i} \pi \delta(x)
$$

and its derivatives [11]. Note that the behaviour of the Feynman Green's function is completely different in even and odd dimensions. Specially, the support of its real part is the wave cone for odd space dimensions, while for even space dimensions inside the wave cone is also part of the support.

The case $D=1$ should be considered separately. for $D=1$, the relations (6.8) and (6.9) are modified to

$$
G_{\mathrm{E}}(t, x)=\frac{1}{4 \pi} \ln \frac{x^{2}+t^{2}}{a^{2}}, \quad D=1
$$

and

$$
G_{\mathrm{F}}(t, x)=\frac{i}{4 \pi} \ln \frac{x^{2}-t^{2}+\mathrm{i} \varepsilon}{a^{2}}, \quad D=1,
$$

where $a$ is an arbitrary constant. So,

$$
\operatorname{Re}\left[G_{\mathrm{F}}(t, x)\right]=-\frac{1}{4} \theta\left(t^{2}-x^{2}\right), \quad D=1,
$$


and

$$
\operatorname{Im}\left[G_{\mathrm{F}}(t, x)\right]=\frac{1}{4 \pi} \ln \left|\frac{x^{2}-t^{2}}{a^{2}}\right|, \quad D=1 .
$$

One then finds the retarded and advanced Green's functions in various dimensions:

$$
G_{\mathrm{R}}(t, \boldsymbol{r})=-2(-1)^{D / 2} \mathrm{pf}\left[\frac{\left(t^{2}-r^{2}\right)^{(1-D) / 2}}{(1-D) S_{D}} \theta\left(t^{2}-r^{2}\right)\right] \theta(t), \quad(D / 2) \in \mathbb{Z},
$$

and

$$
G_{\mathrm{R}}(t, \boldsymbol{r})=-\frac{1}{2 \pi^{(D-1) / 2}} \theta^{[(D-1) / 2]}\left(t^{2}-r^{2}\right) \theta(t), \quad[(D-1) / 2] \in \mathbb{Z} .
$$

The advanced Green's function is the same as the retarded Green's function, with the substitution of $\theta(-t)$ instead of $\theta(t)$.

\section{CONCLUDING REMARKS}

The Green's functions of the wave equation in various dimensions were discussed. It was shown that there are various kinds of Green's functions. Among these Green's functions, the relation of the Feynman Green's with the Green's function of the Laplace equation was found to be particularly simple: these two are simply related to each other through an analytic continuation of the time coordinate to imaginary values. Using this, the retarded and advanced Green's functions were also obtained. An interesting result is that while for odd space dimensions greater than one the support of these Green's functions is on the wave cone, for even space dimensions the support of these Green's functions contains inside the wave cone as well. As a result, for odd space dimensions greater than one the effect of a point source in space time is felt at any point of the space only at a single instant, while for even space dimensions the effect of a point source in space time is felt at any point of the space from a specific time and is continued afterwards.

\section{ACKNOWLEDGEMENTS}

This work was supported by the research council of the Alzahra University.

\section{REFERENCES}

[1] F. B. Byron and R. W. Fuller, Mathematics of classical and quantum physics. Dover, 1992.

[2] D. J. Griffiths, Introduction to electrodynamics. Prentice Hall, 1989.

[3] C. Itzykson and J. B. Zuber, Quantum field theory. McGraw-Hill, 1988.

[4] J. D. Jackson, Classical electrodynamics. John Wiley \& Sons, 1999.

[5] B. Lautrup, Physics of continuous matter: exotic and everyday phenomena in the macroscopic world. IOP Publishing, 2005.

[6] P. M. Morse and K. U. Ingard, Theoretical acoustics. McGraw-Hill, 1968.

[7] S. Nettel, Wave physics: oscillations, solitons, chaos. Springer, 2003. 
[8] S. Polidoro and M. A. Ragusa, "Sobolev-Morrey spaces related to an ultraparabolic equation." Manuscripta Math., vol. 96, no. 3, pp. 371-392, 1998, doi: 10.1007/s002290050072.

[9] S. Polidoro and M. A. Ragusa, "Hölder regularity for solutions of ultraparabolic equations in divergence form.” Potential Anal., vol. 14, no. 4, pp. 341-350, 2001, doi: 10.1023/A:1011261019736.

[10] W. Rindler, Essential relativity: special, general, and cosmological. Springer, 1986.

[11] I. Stackgold, Green's functions and boundary value problems. John Wiley \& Sons, 1998.

[12] W. A. Strauss, Partial differential equations: an introduction. John Wiley \& Sons, 1992.

[13] M. E. Taylor, Partial differential equations I. Springer, 2011. doi: 10.1007/978-1-4419-7055-8.

\section{Author's address}

\section{Mohammad Khorrami}

Department of Physics, Faculty of Physics and Chemistry, Alzahra University, Tehran, Iran

E-mail address: mamwadealzahra.ac.ir 